\title{
DIFFERENTIABLE FUNCTIONS, FORMAL POWER SERIES, AND MOMENTS
}

\author{
H. MIRKIL
}

Every maximal ideal in the algebra $\mathfrak{D}^{\infty}$ of infinitely-differentiable functions on an $n$-dimensional compact manifold $x_{n}$ is of the form $M(x)=\left\{f \in D^{\infty}: f(x)=0\right\}$ for some fixed $x \in X_{n}$. The smallest primary ideal in $M(x)$, i.e. the smallest ideal contained in no maximal ideal other than $M(x)$, is $J(x)=\left\{f \in D^{\infty}: f \equiv 0\right.$ on some neighborhood $V_{f}$ of $x\}$. These two facts more or less exhaust our purely algebraic knowledge of the ideals in $D^{\infty}$. But $D^{\infty}$ has also a natural complete metric vector space topology under which a series $\sum f_{k}$ is said to converge if for each differential operator $D$ the derived series $\sum D f_{k}$ converges uniformly. The closure of $J(x)$ in this topology is the smallest closed primary ideal in $M(x)$, and every closed ideal is the intersection of closed primary ideals. See Whitney [4], Schwartz [3]. $\bar{J}(x)$ can be described concretely as the $f$ that vanish together with all derivatives at $x$. (And most sophomore calculus students are convinced that $\bar{J}(0)$ contains precisely two elements: $e^{-1 / x^{2}}$ and 0 .)

If $h_{1}, \cdots, h_{n} \in M(x)$ are local coordinate functions on $x_{n}$, then their images $X_{1}, \cdots, X_{n}$ generate the quotient algebra $D^{\infty} / \bar{J}(x)$ and the mapping $D^{\infty} \rightarrow D^{\infty} / \bar{J}(x)$ sends every $f$ into its formal taylor series expansion in these coordinates. The following proposition shows that $D^{\infty} / \bar{J}(x)$ is actually the algebra of all formal power series in $X_{1}, \cdots$, $X_{n}$. The one-dimensional case was proved by E. Borel [1] half a century ago, and recently re-proved by Rosenthal [2]. Although the $n$-dimensional case is well-known to persons interested in the behavior of derivatives, it seems never to have been written down publicly. And the present proof has some merit even for $n=1$.

PROPOSITION 1. Let $\lambda_{p_{1}} \ldots p_{n}$ be an arbitrary family of complex numbers, indexed by $n$-tuples $\left(p_{1}, \cdots, p_{n}\right)$ of non-negative integers. Then there exists some infinitely-differentiable function $f$ of $n$ real variables that has as its derivatives at the origin exactly these complex numbers.

$$
\left(\frac{\partial}{\partial \xi_{1}}\right)^{p_{1}} \cdots\left(\frac{\partial}{\partial \xi_{n}}\right)^{p_{n}} f(0, \cdots, 0)=\lambda_{p_{1} \cdots p_{n}} .
$$

It will be convenient to make $n$-dimensional statements in a onedimensional notation. For any $n$-tuple $P=\left(p_{1}, \cdots, p_{n}\right)$ define

Received by the editors August 19, 1955. 
$|P|=p_{1}+\cdots+p_{n}$. For any point $x=\left(\xi_{1}, \cdots, \xi_{n}\right)$ in real $n$-space and any $f \in D^{\infty}$ write $f^{(P)}(x)$ instead of

$$
\left(\frac{\partial}{\partial \xi_{1}}\right)^{p_{1}} \cdots\left(\frac{\partial}{\partial \xi_{n}}\right)^{p_{n}} f\left(\xi_{1}, \cdots, \xi_{n}\right) .
$$

We are looking for an $f$ that satisfies all the conditions

$$
f^{(P)}(0)=\lambda_{P} \text {. }
$$

Since we are concerned only with what happens near the origin, every $f$ that appears in our calculations will be assumed to vanish for $|x| \geqq 1$. Write $\|f\|$ for $\sup _{x}|f(x)|$.

Proof of Proposition 1. We shall construct by induction a sequence $f_{k}$ of functions satisfying

$$
\begin{aligned}
& f_{0}^{(P)}(0)+\cdots+f_{k}^{(P)}(0)=\lambda_{P}, \quad \text { for }|P|=k, \\
& f_{k}^{(P)}(0)=0, \quad \text { for }|P|<k, \\
& \left\|f_{k}^{(P)}\right\|<2^{-k}, \quad \text { for }|P|<k .
\end{aligned}
$$

We can then define $f=\sum f_{k}$. The series will converge in $D^{\infty}$ by condition (3), and its derivatives can be evaluated term by term, giving $f^{(P)}(0)=f_{0}^{(P)}(0)+\cdots+f_{|P|}^{(P)}(0)+\sum_{k>|P|} f_{k}^{(P)}(0)=\lambda_{P}+0$, Q.E.D.

To construct $f_{0}$ is trivial. And given $f_{0}, \cdots, f_{k-1}$ then an $f_{k}$ satisfying (1) and (2) can be manufactured by multiplying a suitable polynomial times a function $\equiv 1$ near the origin and $\equiv 0$ for $|x| \geqq 1$. If now $f_{k}$ does not satisfy (3) replace it by $\rho^{-k} f_{k}(\rho x)=\rho^{-k} f_{k}\left(\rho \xi_{1}, \cdots, \rho \xi_{n}\right)$ with some large positive $\rho$. The new $f_{k}$ still satisfies (1) and (2) and has $\left\|f_{k}^{(P)}\right\|$ as small as we like for $|P|<k$.

With the help of various series and integral transforms, Proposition 1 can be used to solve moment problems. Proposition 2 below is offered as a sample. It is exactly the fourier transform of Proposition 1 , except that the family $\lambda_{P}$ must be modified to compensate for multiplication by $i^{|P|}$. Recall that the transform of a compact-supported function is analytic, and that for every $m>0$ the transform of an infinitely-differentiable function vanishes faster at infinity than $|x|-m$.

PROPOSITION 2. Given an arbitrary family $\lambda_{p_{1}} \ldots p_{n}$ of complex numbers indexed by $n$-tuples of non-negative integers, there exists an analytic function $f$ whose $\left(p_{1}, \cdots, p_{n}\right)$ th moment is $\lambda_{p_{1}} \cdots p_{n}$ for each $\left(p_{1}, \cdots\right.$, $\left.p_{n}\right)$. 


$$
\int \cdots \int f\left(\xi_{1}, \cdots, \xi_{n}\right) \xi_{1}^{p_{1}} \cdots \xi_{n}^{p_{n}} d \xi_{1} \cdots d \xi_{n}=\lambda_{p_{1}} \cdots p_{n} .
$$

All integrals are absolutely convergent.

\section{REFERENCES}

1. E. Borel, Sur quelques points de la theorie des fonctions, Ann. Ecole Norm. (3) vol. 12 (1895) p. 44.

2. A. Rosenthal, On functions with infinitely many derivatives, Proc. Amer. Math. Soc. vol. 4 (1953) pp. 600-602.

3. L. Schwartz, Analyse et synthèse harmoniques dans les espaces de distributions, Canadian Journal of Mathematics vol. 3 (1951) p. 506.

4. $\mathrm{H}$. Whitney, On ideals of differentiable functions, Amer. J. Math. vol. 70 (1948) pp. 634-658.

Dartmouth College 